University of Wollongong

Research Online

Faculty of Social Sciences - Papers (Archive) Faculty of Arts, Social Sciences \& Humanities

2014

Nontarget-to-nontarget interval determines the nontarget P300 in an auditory equiprobable Go/NoGo task

Genevieve Z. Steiner

University of Wollongong, gsteiner@uow.edu.au

Robert Barry

University of Wollongong, rbarry@uow.edu.au

Craig J. Gonsalvez

University of Wollongong, craigg@uow.edu.au

Follow this and additional works at: https://ro.uow.edu.au/sspapers

Research Online is the open access institutional repository for the University of Wollongong. For further information contact the UOW Library: research-pubs@uow.edu.au 


\title{
Nontarget-to-nontarget interval determines the nontarget P300 in an auditory equiprobable Go/NoGo task
}

\author{
Abstract \\ Increases in the target-to-target interval (TTI) systematically enhance the amplitude of the target P300 \\ ERP component. Research examining changes in nontarget P300 related to nontarget-to-nontarget \\ interval (NNI) or sequential probability manipulations has produced inconsistent results, with some \\ studies reporting no enhancement in nontarget P300 and others finding response profiles analogous to \\ TTI effects. Our aim was to clarify these differences. All participants completed a specially designed \\ auditory equiprobable Go/NoGo task with manipulations of TTI and NNI while their EEG activity was \\ recorded. P300 amplitudes were extracted using temporal PCA with Varimax rotation. P3b to targets and \\ nontargets increased systematically as respective TTIs/NNIs increased, but this change did not differ \\ between stimulus types. The Slow Wave did not show any effect of interval, but was more positive to \\ targets than nontargets when interval was collapsed. P3b findings show that matching-stimulus interval \\ effects are not restricted to targets, but discrepancies relative to previous research suggest that NNI \\ effects in P3b may depend on additional processing of nontarget stimuli.

\section{Keywords} \\ determines, task, nogo, p300, auditory, go, equiprobable, nontarget, interval

\section{Publication Details} \\ Steiner, G. Z., Barry, R. J. \& Gonsalvez, C. J. (2014). Nontarget-to-nontarget interval determines the \\ nontarget P300 in an auditory equiprobable Go/NoGo task. International Journal of Psychophysiology, 92 \\ (3), 113-121.
}


Nontarget-to-nontarget interval determines the nontarget P300 in an auditory equiprobable Go/NoGo task

Genevieve Z. Steiner*, Robert J. Barry, \& Craig J. Gonsalvez

Centre for Psychophysics, Psychophysiology, and Psychopharmacology; Brain \& Behaviour Research Institute and School of Psychology, University of Wollongong, Wollongong NSW 2522, Australia

Note to type-setter: "Brain \& Behaviour Research Institute” is a registered name and should not be changed in any way.

*Corresponding author

Email: genevieve_steiner@uow.edu.au

Phone: +61 242215547 


\begin{abstract}
Increases in the target-to-target interval (TTI) systematically enhance the amplitude of the target P300 ERP component. Research examining changes in nontarget P300 related to nontarget-to-nontarget interval (NNI) or sequential probability manipulations has produced inconsistent results, with some studies reporting no enhancement in nontarget P300 and others finding response profiles analogous to TTI effects. Our aim was to clarify these differences. All participants completed a specially designed auditory equiprobable Go/NoGo task with manipulations of TTI and NNI while their EEG activity was recorded. P300 amplitudes were extracted using temporal PCA with Varimax rotation. P3b to targets and nontargets increased systematically as respective TTIs/NNIs increased, but this change did not differ between stimulus types. The Slow Wave did not show any effect of interval, but was more positive to targets than nontargets when interval was collapsed. P3b findings show that matching-stimulus interval effects are not restricted to targets, but discrepancies relative to previous research suggest that NNI effects in P3b may depend on additional processing of nontarget stimuli.
\end{abstract}

Keywords: Event-related potentials (ERPs); Target-to-target interval (TTI); Nontarget-tonontarget interval (NNI); Sequence effects; Interstimulus interval (ISI); P300; Late Positive Complex (LPC); P3b; Slow Wave (SW); LORETA 


\section{Introduction}

The P300 is a late positive deflection in the event-related potential (ERP) with a centroparietal scalp distribution (Picton, 1992; Pritchard, 1981; Sutton, 1965). Underlying this late positive complex are several independent components (P3a, P3b, Novelty P3, Slow Wave; SW), that differ in their latency, topography, and responsivity to task demands (Courchesne et al., 1975; Ritter et al., 1968; Squires et al., 1975; Vaughan \& Ritter, 1970). P300 has been extensively examined as an electrophysiological correlate of information processing (Donchin et al., 1983; Polich, 2007), with early studies focussing on P300 elicitation in oddball task variants (e.g., Duncan-Johnson \& Donchin, 1977).

Within the oddball and Go/NoGo context, P300 amplitude and latency, and reaction time (RT), vary with stimulus presentation characteristics (Gonsalvez et al., 1999). Early studies consistently reported P300 component magnitude changes related to several factors, including: global probability (Duncan-Johnson \& Donchin, 1977; Polich \& Bondurant, 1997; Polich et al., 1991), stimulus sequence (Johnson \& Donchin, 1982; Leuthold \& Sommer, 1993; Sams et al., 1983, 1984; Squires et al., 1976, 1977; Starr et al., 1997; Verleger, 1987), and interstimulus interval (ISI; Fitzgerald \& Picton, 1981; Miltner et al., 1991; Polich 1990a, b). However, changes in these variables also alter the temporal probability of the target, that is, the probability of a target over a period of time, and importantly this changes the target-totarget interval (TTI), the interval between instances of target stimuli.

The importance of temporal probability in determining P300 amplitude was illustrated in Fitzgerald and Picton (1981), where ISI and stimulus sequence were manipulated whilst temporal probability was held constant. It was demonstrated that changes in amplitude to later components in the ERP, including P300 and SW, were related to temporal probability and unrelated to sequential probability and temporal uncertainty. This effect was contingent upon the stimulus being considered a target, where P300 and SW were not elicited by 
standard nontarget stimuli, regardless of their probability. Within the framework proposed by Squires et al. (1976, 1977), Fitzgerald and Picton (1981) suggested that the amplitude of the P300 was, at least partially, related to the decay of a memory trace that is determined by temporal factors.

In a later series of studies by Gonsalvez and colleagues (Croft et al., 2003; Gonsalvez et al., 1999, 2007; Gonsalvez \& Polich, 2002; Steiner et al., 2013b), it was reliably demonstrated that when probability, sequence, and ISI are controlled, TTI emerges as an independent factor determining P300 amplitude and latency. In line with Fitzgerald and Picton (1981), this again suggested that temporal factors, rather than sequence and probability, are important in determining the P300. Gonsalvez et al.'s studies all reported that as TTI increased, P300 amplitude increased and latency decreased.

The TTI effect on RT is not as consistent as with the P300 (and underlying components), as both increases and decreases have been reported across a range of paradigms. For example, Gonsalvez et al. (2007) reported an increase in RT over TTIs, where Steiner et al. (2013b) reported a decrease. This suggests different (or perhaps overlapping) mechanisms of the P300 and RT; a dissociation frequently reported in the literature (e.g., Matt et al., 1992; Sommer et al., 1990; Verleger, 1997; Verleger et al., 2005). For instance, Verleger et al. (2005) suggests that P3b reflects a monitoring process linking stimulus classification and response initiation.

One current perspective on the mechanism of TTI effects on the P300 is the templateupdate model, where TTI effects index an ongoing process of encoding, update, and decay of an immediate memory trace (template) of a stimulus (Gonsalvez et al., 2007; Steiner et al., 2013a). Here, a template is a profile of neural activation representing a stimulus, or stimulusresponse relationship, rather than a specific structure of events (context) within a sequence (as noted by Squires et al., 1976), or an unexpected (Donchin \& Coles, 1988) or awaited 
event (Verleger, 1988). One question arising from this model is whether TTI effects are only elicited by a stimulus associated with a response (target), or whether a simple nontarget can evoke a similar P300 response profile.

Stimulus sequence studies suggest that the nontarget-to-nontarget interval (NNI) affects P300 measures similarly to TTI (Duncan-Johnson \& Donchin, 1977; Hermanutz et al., 1981; Johnson \& Donchin, 1980; Sams et al., 1983; Squires et al., 1977; Starr et al., 1997; Verleger, 1987). For example, Hermanutz et al. (1981) manipulated serial position, and showed that P300 amplitude to rare targets increased as serial position increased, however, the P300 to frequent standards showed a similar pattern: after an initial decrease, a steady increase with serial position was observed. This finding ran counter to the authors' predictions, and was partly attributed to the 'special nature' of the task “i.e. the lengths of the sequences used, their equiprobability, or some other aspect of our constraints on randomness" (p. 421). However, other studies from different laboratories using equiprobable tasks have reported similar results (e.g., Johnson \& Donchin, 1980; Squires et al., 1977; Verleger, 1987). For instance, Squires et al. (1977) reported larger P300s to nontargets when preceded by longer, rather than shorter, chains of targets (i.e., TTTTN $>$ TTTN $>$ TTN $>$ TN); this was replicated in Verleger (1987) for nontargets (i.e., TTN > NNN). In their two-stimulus task, Johnson and Donchin (1980) reported a similar pattern (i.e., TTTTN > TTTN > TTN > TN), and in their three-stimulus task P300 amplitudes were enhanced to nontargets when preceded by longer, compared to shorter, chains of a different nontarget (i.e., $\mathrm{N}_{1} \mathrm{~N}_{1} \mathrm{~N}_{1} \mathrm{~N}_{1} \mathrm{~N}_{2}>$ $\mathrm{N}_{1} \mathrm{~N}_{1} \mathrm{~N}_{1} \mathrm{~N}_{2}>\mathrm{N}_{1} \mathrm{~N}_{1} \mathrm{~N}_{2}>\mathrm{N}_{1} \mathrm{~N}_{2}$ ). These studies demonstrate that the effect of sequence length on nontarget P300 amplitude is large. However, exploring sequential probability in this fashion also inadvertently manipulates the NNI, and whether these previous results are an outcome of this requires further exploration. 
Previous research that directly manipulated the NNI has reported a different pattern of results to the stimulus sequence studies described above. In a three-stimulus task with equiprobable targets and infrequent nontargets, Steiner et al. (2013b) reported typical TTI effects in the P300, but this pattern was not replicated for the infrequent NNI. Similarly, in a three-stimulus fMRI study, again with equiprobable targets and infrequent nontargets, Stevens et al. (2005) found TTI effects in hemodynamic activity, but no changes with infrequent-novel NNI. In summary, several studies showed that P300 amplitude increases as NNI increases (Hermanutz et al., 1981; Johnson \& Donchin, 1980; Squires et al., 1977; Verleger 1987), where others reported no change in P300 (Steiner et al., 2013b) or hemodynamic activity (Stevens et al., 2005). The reason for these inconsistent result patterns requires clarification.

Some of the discrepancies in the results described above may have resulted from broad differences in the designs of these conflicting studies, such as the matching-stimulus interval content, similarities/differences between multiple nontargets, or relative target/frequent standard probability. For instance, some studies interspersed infrequent and novel NNIs with standards and targets (e.g., $\mathrm{N}_{1} \mathrm{~N}_{2} \mathrm{TN}_{2} \mathrm{~N}_{1}$; Steiner et al., 2013b; Stevens et al., 2005), where others structured NNI sequences containing only the target (NTTTN) or another nontarget $\left(\mathrm{N}_{1} \mathrm{~N}_{2} \mathrm{~N}_{2} \mathrm{~N}_{2} \mathrm{~N}_{1}\right.$; Johnson \& Donchin, 1980; Squires et al., 1977; Verleger, 1987). Additionally, some studies employed tasks where the nontarget of interest had a lower probability than the frequent standard (e.g., Hermanutz et al., 1981: target $\mathrm{p}=.10$, infrequent $=.10$, standard $\mathrm{p}=.80$; Steiner et al., 2013b: target $\mathrm{p}=.25$, infrequent $\mathrm{p}=.25$, standard $\mathrm{p}=$ .50 ; Stevens et al., 2005: target $\mathrm{p}=.10$, novel $\mathrm{p}=.10$, standard $\mathrm{p}=.80$ ), where others used equiprobable two- (Johnson \& Donchin, 1980; Verleger, 1987: target $\mathrm{p}=.50$, nontarget $\mathrm{p}=$ .50) and three-stimulus (Johnson \& Donchin, 1980; Squires et al., 1977: target p = .33, nontarget $_{1} \mathrm{p}=.33$, nontarget $2 \mathrm{p}=.33$ ) tasks. The differences in the designs of these studies, 
and the relative discrepancies in their findings, highlight the need for a careful and methodical approach to testing whether matching-stimulus interval effects are apparent for nontargets. In addition, some of those studies focused on baseline-to-peak derived P300 measures, and, as a result, may have overlooked possible NNI effects in underlying P300 components (e.g., P3a, P3b, SW etc.).

The current study aimed to resolve the differences in NNI effects reported above by clarifying whether nontargets elicit interval effects on components of the P300 akin to those reported for targets. A specific paradigm was carefully constructed to test this aim: an auditory equiprobable ${ }^{1}$ Go/NoGo task containing targets and nontargets at various TTIs and NNIs that were interspersed with silence to control global probability. Consistent with previous TTI studies (Croft et al., 2003; Gonsalvez et al., 1999, 2007; Gonsalvez \& Polich, 2002; Steiner et al., 2013a, b), as TTI increased, we expected P3b and SW amplitudes to increase, and RTs to increase then decrease. It was also predicted that without the interference of an additional nontarget being presented within NNIs, NNI effects would be seen in at least one component of the P300 complex (consistent with Hermanutz et al., 1981; Johnson \& Donchin, 1980; Squires et al., 1977; Verleger, 1987). We were also interested in whether the TTI, and possibly NNI, mechanism affected different components of the P300 (e.g., P3b vs. SW), and whether this would differ between stimulus types.

\section{Method}

\subsection{Participants}

Twenty University of Wollongong undergraduates (mean age $=19.9$ years, $S D=1.9$; 11 males, 18 right-handed) participated in return for course credit. All provided informed consent prior to commencing the experiment, and were free to withdraw at any time without

\footnotetext{
${ }^{1}$ An equiprobable task was utilised specifically to ensure that any differences in P300 between stimulus types were related to task relevance.
} 
penalty. Individuals taking psychotropic medication, and those with self-reported neurological or psychiatric illnesses, were excluded. Self-reports indicated that participants had refrained from psychoactive substances for at least 12 hours and from tea, coffee, alcohol, and cigarettes for at least 2 hours prior to testing. All participants had normal or corrected-to-normal vision and self-reported normal hearing.

\subsection{Procedure}

After providing informed consent, participants completed a demographic and screening questionnaire, and were fitted with EEG recording apparatus. Prior to the experiment, participants completed an electrooculogram (EOG)/EEG calibration task (Croft \& Barry, 2000). Participants were seated in an air-conditioned room 600-800 mm in front of a $48.3 \mathrm{~cm}$ (19”) Dell LCD monitor and instructed to fixate on a $10 \times 10 \mathrm{~mm}$ grey cross centred on a black background. Acoustic stimuli were delivered binaurally through Sony MDR V700 circumaural stereo headphones, and consisted of 1000 and $1500 \mathrm{~Hz}$ tones of 50 ms duration (15 ms rise/fall time), at $60 \mathrm{~dB}$ SPL.

The experiment consisted of a unique task ${ }^{2}$ that was broken into four different blocks (approximately 4.5 min each), with short rest intervals between blocks to minimise fatigue. To avoid global probability effects, the task was an equiprobable oddball (Go/NoGo) paradigm (global $\mathrm{p}=0.50$ ), where target and nontarget stimuli were counterbalanced 1000 and $1500 \mathrm{~Hz}$ tones (across participants). When designing the paradigm, the presentation of TTIs and NNIs was randomised and silence was added to maintain stimulus equiprobability (Figure 1). That is, desired TTIs and NNIs were obtained by varying the stimulus onset asynchrony (SOA). Care was taken to vary the local probability and density of targets, nontargets, and silence to minimise possible expectancy effects related to sequence and ISI.

\footnotetext{
${ }^{2}$ It should be noted that this paradigm is based on that used in Steiner et al. (2013a), but here we added a manipulation of NNI.
} 
The stimulus order was fixed across subjects, with specific presentations of eight TTIs/NNIs (1, 2, 3, 5, 7, 10, 12, 15 s); a total of 264 trials were presented over the 4 blocks (132 targets; 132 nontargets). Exactly 20 trials for each of the five intervals of major interest (1, 2, 5, 10, 15 s) were presented with equal probability. Thus, stimulus order was semi-random with a variable SOA that was no less than $1 \mathrm{~s}$ for successive stimuli (TT, NN, TN, NT). Between presentations of matching stimuli, for intervals longer than $1 \mathrm{~s}$, there was silence or the other stimulus type (or a combination of the two for intervals $>2 \mathrm{~s}$ ). To balance possible speed/accuracy trade-offs, participants were instructed to "respond to target stimuli with a button press, as quickly and as accurately as possible”. Participants responded with their dominant hand on a Logitech ${ }^{\circledR}$ Precision game controller. Instruction was given to sit as still as possible, but participants were not directly instructed to refrain from blinking (Verleger, 1991). This procedure was approved by the joint South Eastern Sydney/Illawarra Area Health Service and University of Wollongong Health and Medical Human Research Ethics Committee.

Figure 1 about here...

\subsection{Materials and Apparatus}

EEG data were recorded continuously using a $70 \mathrm{~Hz}$ lowpass filter from A2 and 30 scalp sites (Fp1, Fp2, F7, F3, Fz, F4, F8, FT7, FC3, FCz, FC4, FT8, T7, C3, Cz, C4, T8, TP7, CP3, CPz, CP4, TP8, P7, P3, Pz, P4, P8, O1, Oz, O2) with an electrode cap using tin electrodes. A1 was used as a reference and the cap was grounded by an electrode located midway between Fp1, Fp2 and Fz. Data were acquired using a Neuroscan Synamps 2 digital signal-processing system and Neuroscan 4.3.1 Acquire software, and were recorded DC - 70 Hz. The display and stimulus markers were controlled by a linked stimulus computer using Neurobehavioral Systems Inc. Presentation V 13.0 Build 01.23.09 software. 
EOG was recorded using tin cup electrodes placed $2 \mathrm{~cm}$ above and below the left eye for vertical movements, and on the outer canthus of each eye for horizontal movements. Impedance was less than $5 \mathrm{k} \Omega$ for cap, EOG, and reference electrodes. Scalp and EOG potentials were amplified with a gain of 500 and digitised at a rate of $1000 \mathrm{~Hz}$.

\subsection{Data Extraction}

Trials containing omission (misses) or commission (false alarms) errors, or response times longer than $800 \mathrm{~ms}$, were excluded. All participants responded accurately to at least 95 $\%$ of trials. The EEG data were EOG corrected using the RAAA EOG Correction Program (Croft \& Barry, 2000), and re-referenced to digitally linked ears in Neuroscan. Single trials were extracted offline using the Neuroscan Edit software, low pass filtered $(0.1-30 \mathrm{~Hz}$, zero-phase shift, $24 \mathrm{~dB}$ /Octave), epoched for $-100 \mathrm{~ms}$ pre- to $750 \mathrm{~ms}$ post-stimulus, and baselined to the pre-stimulus period. Data were manually inspected for any additional artefact; any contaminated trials were rejected. For each stimulus type, averages were computed for each subject for each of the five intervals of major interest.

\subsection{Principal Components Analysis}

The averaged data (-100 to 750 ms: 850 datapoints) from 30 scalp locations were submitted to a temporal PCA using Dien’s ERP PCA toolkit (v. 2.23; Dien, 2010) in MATLAB (The Mathworks, R14SP3). Data for the PCA were half-sampled to 425 timepoints (variables) to reduce computation time. Factors for all conditions were quantified simultaneously (6000 observations: 20 participants $\times 2$ stimulus types $\times 5$ intervals $\times 30$ sites). The PCA used the unstandardised covariance matrix with Kaiser normalisation, and all 425 unrestricted factors underwent Varimax rotation, following Kayser and Tenke (2003). PCA factors were identified as ERP components based on their latency, topography, and polarity of their conspicuous maximum loading. Although this quantification procedure was performed for all components poststimulus, this study was interested in testing hypotheses 
pertaining to the P300, thus only components identifiable as part of the P300/late positive complex were retained for analysis. The factor scores for these components were output and entered into subsequent statistical analyses.

\subsection{Statistical Analyses}

To define the topography for each identified P300 component, separate MANOVAs were carried out on the microvolt-scaled factor scores (Dien, 2012) at the 9 inner sites (F3, Fz, F4, C3, Cz, C4, P3, Pz, P4), pooled across both stimulus types and the five interval levels. It should be noted that although only 9 sites were analysed, the PCA was conducted on all recording sites as all scores were needed to produce topographic headmaps. The topographic examination involved the sagittal plane: Frontal (F3, Fz, F4), central (C3, Cz, C4), and parietal (P3, Pz, P4); and the coronal plane: Left (F3, C3, P3), midline (Fz, Cz, Pz), and right (F4, C4, P4). Planned contrasts compared regions within each plane: Sagittal plane (frontal vs. parietal, and central vs. mean of the frontal and parietal) and coronal plane (left vs. right, and midline vs. mean of the left and right sites). The topographic distribution of component amplitudes can be examined efficiently by utilising these orthogonal planned contrasts. No Bonferroni-type $\alpha$ adjustment was required as contrasts were planned, and the number of contrasts did not exceed the degrees of freedom for effect (Tabachnick \& Fidell, 1989). Component topography was defined as the mean amplitude across the maximal region (e.g., parietal maximum $\rightarrow$ mean across $\mathrm{P} 3, \mathrm{Pz}$, and $\mathrm{P} 4)$; using a mean across a region defined by multiple sites, rather than a single electrode, reduces the impact of chance variance at a single site.

eLORETA (exact low resolution brain electromagnetic tomography; Pascual-Marqui, 1999; Pascual-Marqui et al., 1994) was used to examine the sources of each P300 component to the stimulus types. This source examination was based on grand mean components as a complement to the statistical analyses of their scalp topography. 
Separate repeated-measures MANOVAs assessed each P300 component for the effects of Interval (5 levels: 1, 2, 5, 10, 15 s) and Stimulus Type (2 levels: Target vs. Nontarget). Within the interval factor, weighted linear and quadratic trends were assessed. RT to target stimuli was assessed over the five interval levels with a one-way repeatedmeasures MANOVA, again with weighted linear and quadratic contrasts.

To assess the difference between RT and P300 response patterns, we followed Nieuwenhuis et al. (2011). These measures were both standardised and their z-scores were assessed with MANOVA with factors of Measure (2 levels: RT and P300 component) and Interval (5 levels: 1, 2, 5, 10, 15 s). The Interval factor again included weighted linear and quadratic trend analyses.

The violations of sphericity assumptions associated with repeated-measures analyses do not affect single degree of freedom contrasts, so Greenhouse-Geisser-type correction was not necessary (O’Brien \& Kaiser, 1985). It should also be noted that, as this paper details results for a number of dependent measures, the frequency of Type I errors increases. However, Howell (1997) argues that adjusting $\alpha$-levels cannot be used to control this increase in frequency of Type I errors. All tests reported have $(1,19)$ degrees of freedom.

\section{Results}

\subsection{Grand Mean ERPs}

Grand mean ERPs from the midline sites are shown for targets and nontargets in the left column of Figure 2. Figure 3 shows the grand mean ERPs to both stimulus types from the midline sites over the five intervals.

Figures 2 and 3 about here...

\subsection{PCA Outcomes}

Of the 425 temporal factors extracted, the first seven explained $86.5 \%$ of the total variance. The right column of Figure 2 displays the sums of these temporal components. 
Comparison with the ERPs in the left panel suggests a good fit with the original data.

The rescaled temporal factor loadings for the seven components are displayed as a function of time in Figure 4 (Tabachnick \& Fidell, 1989). The percentage of variance explained, latency, and factor order for each rotated component are also indicated. The topographic headmaps of these components, averaged across stimulus type and interval, are displayed above. These components were tentatively identified in terms of their polarity, latency, temporal sequence, and topography as N1, Processing Negativity (PN; temporal negativity occurring relatively late in the N1 latency range, described in Näätänen \& Picton, 1987), P2, N2, P3b (distinct parietal positivity), bipolar SW, and a very late negativity (VLN).

Figure 4 about here...

There were two components identifiable as part of the P300 complex: P3b and SW. In the right panel of Figure 2, the sum of these two components is illustrated with dashed lines at Pz. Other than a small deviation from the actual data, starting around $400 \mathrm{~ms}$, a good fit with the P300 (particularly the PCA data) is apparent, indicating that these two components are explaining a substantial proportion of the variance in the P300 complex, and that their analysis is justified. The analyses of these components are reported below in order of component latency. Trends examined as a function of interval are denoted as "linear intervals” or "quadratic intervals”. The direction of effects is written as “<” and ">>, and interactions between variables as “ $\times$ ”.

\subsection{Topography}

The outcome of the topographic analyses, including the $F$ - and $p$-values, and partial effect sizes, are detailed in Table 1 for the two P300 components. As illustrated in Figure 4, grand mean P3b was largest parietally, lower centrally, and larger in the midline. Laterally, P3b was greatest in the right-central regions. P3b was thus defined as parietally maximal 
(i.e., $66 \%$ variance explained by parietal $>$ frontal effect within the sagittal plane), and the mean of these sites (P3, Pz, P4) was selected for further analysis.

SW showed the typical frontally negative/parietally positive scalp distribution (Figure 4), with the difference between frontal and parietal sites being greatest at the midline, and in the left. The frontal/parietal difference explained $74 \%$ of the variance in the SW scalp distribution. Thus, to reflect this typical bipolar topography, SW was defined as the difference between frontal and parietal sites (i.e., [mean P3, Pz, P4] minus [mean F3, Fz, F4]).

Table 1 about here...

\subsubsection{PCA component source localisation}

The eLORETA source plots of P3b and SW are illustrated in Figure 5, separately for targets and nontargets. eLORETA found a number of sources active for these two components; for simplicity, we detail the three major sources for each component and stimulus type. For target P3b, this was BA5: postcentral gyrus, paracentral lobule, and superior parietal lobule. Nontarget P3b was maximal in postcentral gyrus (BA5, 7), superior parietal lobule (BA7), and precuneus (BA7). The major sources for SW to targets were the medial frontal gryus (BA6), cingulate gyrus (BA31), and the paracentral lobule (BA31), and for nontargets, these were in BA6: middle frontal gyrus, superior frontal gyrus, and medial frontal gyrus.

Figure 5 about here...

\subsection{Effects of Interval and Stimulus Type}

\subsubsection{Factor 3: P3b}

P3b demonstrated a main effect of interval, with amplitude increasing in a linear fashion as interval increased (across both TTI and NNI; linear intervals: $F=30.07, p<.001$, $\eta_{p}{ }^{2}=.61$; Figure 6, top panel). This increase in P3b amplitude plateaued from around $10 \mathrm{~s}$ 
(quadratic intervals: $F=13.45, p=.002, \eta_{p}{ }^{2}=.41$ ). There was no main effect of stimulus type $\left(F=1.03, p=.324, \eta_{p}{ }^{2}=.05\right)$ and no interval $\times$ stimulus type interaction $(F=.31, p=$ $\left..585, \eta_{p}^{2}=.02\right)$.

Figure 6 about here...

\subsubsection{Factor 2: SW}

The frontally negative/parietally positive SW did not demonstrate a main effect of interval (Figure 6, middle panel; $F=.37, p=.550, \eta_{p}{ }^{2}=.02$ ). As shown in Figure 6, there was a main effect of stimulus type, where the frontally negativity/parietally positive difference was more positive for targets than nontargets (frontal $<$ parietal $\times$ target $>$ nontarget: $\left.F=40.90, p<.001, \eta_{p}{ }^{2}=.68\right)$. There was no interval $\times$ stimulus type interaction $\left(F=2.99, p=.100, \eta_{p}^{2}=.14\right)$.

\subsubsection{RT}

The bottom panel of Figure 6 shows mean RT as a function of TTI. RT increased up to TTI-5 s before decreasing (quadratic intervals: $F=18.31, p<.001, \eta_{p}{ }^{2}=.49$ ). The RT and P3b comparison produced a statistically significant measure $\times$ linear interval interaction $(F=$ 4.88, $p<.040, \eta_{p}{ }^{2}=.20$ ), indicating that TTI effects were more systematic for P3b than RT.

\section{Discussion}

We investigated the matching-stimulus interval/P300 relationship in a speciallydesigned auditory equiprobable Go/NoGo task with varying TTIs/NNIs. Our TTI findings were as expected, with longer TTIs producing larger P3b target responses, and RT showing an initial increase to shorter TTIs (TTI $<5$ s) and a decrease to longer TTIs. Importantly, the increase in P3b did not differ between TTI and NNI. SW, however, did not show the same pattern of results; this is discussed further below.

Seven components were identified from the PCA including N1, PN, P2, N2, P3b, SW, and a VLN, and were mostly comparable with other equiprobable studies (e.g., Barry \& De 
Blasio, 2013). Verleger and Möcks (1987) note that slow waves, such as the large, a-typical VLN, may arise from the misallocation of variance due to data not returning to baseline within the Varimax-rotated span. However, a follow up analysis showed that the VLN, the target/nontarget differences, and the ERP resolved by $850 \mathrm{~ms}$ poststimulus. This, together with the ERP morphology, suggests VLN is a real component; further research is required to confirm its validity.

The PCA-derived ERPs were largely similar to the original data (Figure 2), as was the reconstructed P300, other than a slight deviation at around 400 ms poststimulus. However, PCA did not produce any other conspicuous components explaining more than $1 \%$ total variance within the P300 range. A slight deviation is potentially noise (in the original data) extracted by the PCA as smaller factors and will not be considered further.

Sources identified for the P3b and SW components were similar to previous research using oddball (Saletu et al., 2008; Volpe et al., 2007) and Go/NoGo tasks (Bokura et al., 2001), there is limited relevant LORETA data from equiprobable Go/NoGo tasks. In Barry and Rushby (2006), LORETA sources identified to Go stimuli were cuneus, cingulate gyrus, and precuneus, and to NoGo, the cingulate gyrus, cuneus, and anterior cingulate. There is some overlap between regions (e.g., precuneus, cingulate gyrus) and similarities with corresponding Brodmann areas (BA7, 31), however, discrepancies could be related to computational differences between LORETA versions. Further research is needed to clarify component sources in equiprobable tasks, and reconcile those findings with other measures (e.g., fMRI and lesion studies).

\subsection{Effects of Interval and Stimulus Type}

Across stimulus type, P3b systematically increased in positivity over matchingstimulus intervals, before plateauing at around the $10 \mathrm{~s}$ interval. Importantly, there was no interaction between interval and stimulus type, indicating that interval effects are not isolated 
to target stimuli. This finding is in line with P300 research examining nontarget sequence effects (Duncan-Johnson \& Donchin, 1977; Hermanutz et al., 1981; Johnson \& Donchin, 1980; Sams et al., 1983; Squires et al., 1977; Starr et al., 1997; Verleger, 1987), but runs counter to our previous three-stimulus study exploring P300 changes to the infrequent NNI (Steiner et al., 2013b). For P3b, there was no statistical difference between stimulus type, as found in Squires et al. (1977) and partly in Verleger (1987). Verleger (1987) noted this deviates from other equiprobable studies (Duncan-Johnson \& Donchin, 1977; Johnson \& Donchin, 1982; Pfefferbaum et al., 1985; Squires et al., 1975). It is possible that our nontarget/NNI P3b results are related to further processing of nontarget stimuli. Several possible explanations for this will now be discussed.

Instructing participants to refrain from blinking is common practice in EEG research, but can generate a secondary task, leading to a reduction in P300 amplitude. To reconcile discrepancies discussed in Verleger (1987), Verleger (1991) demonstrated that equiprobable targets do not evoke larger P300s than nontargets when participants are instructed to refrain from blinking. This instruction was not directly provided in the current study, Squires et al. (1977), or Verleger (1987), making this an unlikely explanation for the lack of stimulusrelated differences in P3b reported here.

The importance assigned to nontargets in a particular task can also affect the P300 response profile. Target/standard context can affect stimulus-processing by influencing an attentional set determining how nontargets are processed (task-relevant, background, distracters; Sawaki \& Katayama, 2006). When processed as task-relevant, nontargets elicit a large target-like P300 (Azizian et al., 2006; Watson et al., 2005); a response-pattern evident in the current data (see Figures 2 and 3). When such a classification is made (which could be due to a range of factors including the physical properties of the stimulus, task structure, distractor vs. standard probability, the blinking instructions already discussed), additional 
processing can occur, perhaps rendering nontargets susceptible to interval effects similar to targets. We do not mean to imply that nontargets in the current study were "task relevant", however, the special task included an unpredictable stimulus sequence and highly variable ISI, which may have increased stimulus classification difficulty and/or facilitated additional nontarget processing.

Regarding the discrepancy with our prior NNI study (Steiner et al., 2013b), tasks containing several nontargets may lead to all irrelevant non-novel stimuli being classified as background information. For instance, in their equiprobable three-stimulus task, Johnson and Donchin (1980) reported that the ERP morphology to two nontargets suggested that they were processed as though prior probability was .67 (i.e., as a single nontarget). Results were similar in Steiner et al. (2013b; highly similar standard/nontarget waveforms), however, marginal topographic differences were detected between nontarget types, suggesting different underlying sources. Despite distinct processing requirements between targets/nontargets, setting their probability equal is not sufficient to elicit NNI effects when additional background information exists (or is contained within NNIs), and may lead to all nontargets being processed as irrelevant background material. Further research is required to justify this position.

The SW did not show the same systematic change over intervals as P3b, a finding both consistent (Hermanutz et al., 1981) and inconsistent (Fitzgerald \& Picton, 1981; Starr et al., 1997; Verleger, 1987) with previous research. Temporal probability effects seem to be at least partially contingent on how SW topography is defined. For example, Steiner et al. (2013) reported TTI effects in the form of a complex interval by topography interaction. Some studies reporting sequential probability effects have analysed either a site that is not representative of the bipolar topography (e.g., Cz: Starr et al., 1997), or frontal and positive aspects separately (e.g., Fz and Pz independently: Fitzgerald \& Picton, 1981). In light of 
Dien’s (2012) recent assertion that the two SW “subcomponents” represent only a single component, it may be suboptimal to analyse one aspect. Here, SW was defined as the frontally-negative/parietally-positive difference, and this produced a main effect of stimulus type (targets more positive than nontargets), a finding consistent with prior research (Fitzgerald \& Picton, 1981; Hermanutz et al., 1981; Starr et al., 1997).

Our RT findings were in line with Steiner et al. (2013a), with RT showing an initial increase to TTIs up to $5 \mathrm{~s}$, followed by a decrease to longer TTIs. When compared with P3b over intervals, RT did not demonstrate a similar systematic linear trend. This emphasises the dissociation between RT and P300 amplitude observed in previous studies (e.g., Matt et al., 1992). Where P300 and TTI appear to be intimately linked, consistently increasing together in a linear fashion, RT demonstrates a range of patterns depending on task demand and structure. This could be a combined effect of both automatic and controlled processes, such as sequence generated expectancies (this is detailed further in Sommer et al., 1998), and/or an overlapping mechanism (Verleger et al., 2005).

\subsection{Theoretical Considerations and Future Research Directions}

The mechanism underpinning matching-stimulus interval effects is yet to be elucidated, but theoretical interpretations have been made. Sommer et al. (1998) borrowed the term “passive expectancies” from Kahneman and Tversky (1982), suggesting that P300 probability effects result from an automatic perceptual process sensitive to stimulus frequency. This frequency detector, also outlined in Karis et al. (1983), or a similar temporal mechanism, could be responsible for TTI/NNI effects, and identifying its source (both conceptually and physically in the brain) would aid our understanding of these temporal effects. For instance, Jentzsch and Sommer (2001) reported a frontal P3(00) component sensitive to sequence, and suggested that it originated in the mesial subcortical or cingulated structures and could play a role in response-selection processes. 
From a template update perspective, encoding of nontargets in working memory may have been adequate here to produce NNI effects, but not sufficient in Steiner et al. (2013b), where a similar template may have been utilised for both frequent and infrequent nontargets. Comparatively, consistent elicitation of TTI effects could be due to the added complexity of a stimulus-response element, where the association with a response is also encoded. Here, additional resources are required, possibly greater networks, with greater overall template decay and update. With multiple nontargets, the linear relationship between interval and P300 may be compromised (as seen in Steiner et al., 2013b), which could be because the same, or shared, neural networks are activated for processing any number of nontarget stimuli; meaning a more frequently activated template that will decay less, require less update, and correspond with reduced P300s. In that case, nontarget P300 may be determined by other factors (e.g., ISI and stimulus sequence), rather than by NNI (such as in Johnson \& Donchin, 1980). Again, this is speculative: there are not enough data available currently to permit the exploration of nontarget template differences. Further work is required to expand this theoretical perspective.

Visual inspection of ERPs reveals interval effects in additional components (P2, N2). However, this is not relevant to the research question of the current study (i.e., are NNI effects apparent for the nontarget $P 300$ ?). Specifically, effort was made to clarify discrepancies within P300 research, hence only those components were analysed. Future studies should explore matching-stimulus interval effects in other ERP components, and replicate early work on sequences effects; work in our laboratory is currently addressing this.

In conclusion, we demonstrated that nontarget P3b amplitude increases systematically with NNI increments. Previous work was replicated by finding a similar pattern in P3b to targets with manipulations of TTI. SW, however, did not evidence the interval effects noted as a complex topographic interaction in our previous investigation (Steiner et al., 2013b), but 
did show greater positivity to targets than nontargets. Importantly, our nontarget P3b findings clarify a discrepancy in the literature, and these, together, enhance our understanding of nontarget stimulus processing. Data suggest that the P3b/TTI relationship is robust across a variety of tasks, and when TTIs are interspersed with multiple nontargets. However, NNI effects seem to be apparent only when there is minimal distraction from other nontargets, and the stimuli of interest are not classified as distracting or background information. Future research should clarify the origin of matching-stimulus interval effects in sequential processing (i.e., ERP components preceding the P300 complex) and their locus in the brain. 


\section{References}

Azizian, A., Freitas, A.L., Watson, T.D., Squires, N.K., 2006. Electrophysiological correlates of categorization: P300 amplitude as index of target similarity. Biological Psychology, 71, 278-288.

Barry, R.J., De Blasio, F.M., 2013. Sequential processing in the equiprobable auditory Go/NoGo task: A temporal PCA study. Int J Psychophysiol, 89, 123-127.

Barry, R.J., Rushby, J.A., 2006. An orienting reflex perspective on anteriorisation of the P3 of the event-related potential. Exp Brain Res, 173, 539-545.

Bokura, H., Yamaguchi, S., Kobayashi, S., 2001. Electrophysiological correlates for response inhibition in a Go/NoGo task. Clin Neurophysiol, 112, 2224-2232.

Croft, R.J., Barry, R.J., 2000. Removal of ocular artifact from the EEG: a review. Clinical Neurophysiology, 30, 5-19.

Croft, R.J., Gonsalvez, C.J., Gabriel, C., Barry, R.J., 2003. Target-to-target interval versus probability effects on P300 in one- and two-tone tasks. Psychophysiology, 40, 322-328.

Courchesne, E., Hillyard, S.A., Galambos, R., 1975. Stimulus novelty, task relevance and the visual evoked potential in man. Electroencephalography and Clinical Neurophysiology, 39,131-143.

Dien, J., 2010. The ERP PCA Toolkit: an open source program for advanced statistical analysis of event-related potential data. Journal of Neuroscience Methods, 187, 138.

Dien, J., 2012. Applying principal components analysis to event-related potentials: a tutorial. Developmental Neuropsychology, 37,497-517.

Donchin, E., Coles, M.G.H., 1988. Is the P300 component a manifestation of context updating? Behavioural Brain Sciences, 11, 357-374.

Donchin, E., McCarthy, G., Kutas, M., Ritter, W., 1983. Event-related brain potentials in the study of consciousness. In R.J. Davidson, G.E. Schwartz, D. Shapiro (Eds.), 
Consciousness and self-regulation (Vol.3, pp. 81-121). New York: Plenum.

Duncan-Johnson, C.C., Donchin, E., 1977. On quantifying surprise: The variation of eventrelated potentials with subjective probability. Psychophysiology, 14, 456-467.

Fitzgerald P.G., Picton, T.W., 1981. Temporal and sequential probability in evoked potential studies. Canadian Journal of Psychology, 35, 188-200.

Gonsalvez, C.J., Barry, R.J., Rushby, J.A., Polich, J., 2007. Target-to-target interval, intensity, and P300 from an auditory single-stimulus task. Psychophysiology, 44, 245250.

Gonsalvez, C.J., Gordon, E., Grayson, S., Barry, R.J., Lazzaro, I., Bahramali, H., 1999. Is the target-to-target interval a critical determinant of P3 amplitude? Psychophysiology, 36, 643-654.

Gonsalvez, C.J., Polich, J., 2002. P300 amplitude is determined by target-to-target interval. Psychophysiology, 39, 388-396.

Hermanutz, M., Cohen, R., Sommer, W., 1981. The effects of serial order in long sequences of auditory stimuli on event-related potentials. Psychophysiology, 18, 415-423.

Howell, D., 1997. Statistical methods for psychology (6 ${ }^{\text {th }}$ ed.). Belmont, CA: Thompson Wadsworth.

Jentzsch, I., Sommer, W., 2001. Sequence-sensitive subcomponents of P300: Topographic analyses and dipole source localization. Psychophysiology, 38, 607-621.

Johnson, R., Jr., Donchin, E., 1980. P300 and stimulus categorization: Two plus one is not so different from one plus one. Psychophysiology, 17, 167-178.

Johnson, R., Jr., Donchin, E., 1982. Sequential expectancies and decision making in a changing environment: an electrophysiological approach. Psychophysiology, 19, 183200.

Kahneman, D., Tversky, A., 1982. Variants of uncertainty. Cognition, 11, 143-157. 
Karis, D., Chesney, G.L., Donchin, E., 1983. “. . . 'twas ten to one; And yet we ventured. . . .”: P300 and decision making. Psychophysiology, 20, 260-268.

Kayser, J., Tenke, C.E., 2003. Optimizing PCA methodology for ERP component identification and measurement: theoretical rationale and empirical evaluation. Clinical Neurophysiology, 114, 2307-2325.

Leuthold, H., Sommer, W., 1993. Stimulus presentation rate dissociates sequential effects in event-related potentials and reaction times. Psychophysiology, 30, 510-517.

Matt, J., Leuthold, H., Sommer, W., 1992. Differential effects of voluntary expectancies on reaction times and event-related potentials: Evidence for automatic and controlled expectancies. Journal of Experimental Psychology: Learning, Memory, and Cognition, 18, 810-822.

Miltner, W., Johnson, R., Braun, C., 1991. Auditory and somatosensory event-related potentials. Journal of Psychophysiology, 5, 27-42.

Näätänen, R., Picton, T., 1987. The N1 wave of the human electric and magnetic response to sound: a review and an analysis of the component structure. Psychophysiology, 24, $375-425$.

Nieuwenhuis, S., Forstmann, B.U., Wagenmakers, E., 2011. Erroneous analyses of interactions in neuroscience: a problem of significance. Nature Neuroscience, 14, 11051107.

O’Brien, R.G., Kaiser, M.K., 1985. MANOVA method for analysing repeated measures designs: An extensive primer. Psychological Bulletin, 97, 316-333.

Pascual-Marqui, R. D., 1999. Review of methods for solving the EEG inverse problem. Int J Bioelectromag, 1, 75-86. 
Pascual-Marqui, R.D., Michel, C.M., Lehmann, D., 1994. Low resolution electromagnetic tomography: a new method for localizing electrical activity in the brain. Int $\mathrm{J}$ Psychophysiol, 18, 49-65.

Pfefferbaum, A., Ford, J.M., Weller, B.J., Kopell, B.S., 1985. ERPs to response production and inhibition. EEG Clin Neurophysiol, 60, 423-434.

Picton, T.W., 1992. The P300 wave of the human event-related potential. Journal of Clinical Neurophysiology, 9, 456-479.

Polich, J., 1990a. P300, probability, and interstimulus interval. Psychophysiology, 27, 396403.

Polich, J., 1990b. Probability and inter-stimulus interval effects on the P300 from auditory stimuli. International Journal of Psychophysiology, 10, 163-170.

Polich, J., 2007. Updating P300: An integrative theory of P3a and P3b. Clinical Neurophysiology, 118, 2128-2148.

Polich, J., Bondurant, T., 1997. P300 sequence effects, probability, and interstimulus interval. Physiology \& Behavior, 61, 843-849,

Polich, J., Brock, T., Geisler, M.W., 1991. P300 from auditory and somatosensory stimuli: probability and inter-stimulus interval. International Journal of Psychophysiology, 11, 219-223.

Pritchard, W.S., 1981. Psychophysiology of P300. Psychological Bulletin, 89, 506-540.

Ritter, W., Vaughan, J.H.G., Costa, L.D., 1968. Orienting and habituation to auditory stimuli: A study of short term changes in average evoked responses. Electroencephalography and Clinical Neurophysiology, 25, 550-556.

Saletu, M., Anderer, P., Saletu-Zyhlarz, G.M., Mandl, M., Zeitlhofer, J., Saletu, B., 2008. Event-related potential low-resolution electromagnetic tomography (ERP-LORETA) 
suggests decreased energetic resources for cognitive processing in narcolepsy. Clin Neurophysiol, 119, 1782-1794.

Sams, M., Alho, K., Näätänen, R., 1983. Sequential effects on the ERP in discriminating two stimuli. Biological Psychology, 17, 41-58.

Sams, M., Alho, K., Näätänen, R., 1984. Short-term habituation and dishabituation of the mismatch negativity of the ERP. Psychophysiology, 21, 434-441.

Sawaki, R., Katayama, J., 2006. Stimulus context determines whether non-target stimuli are processed as task-relevant or distractor information. Clinical Neurophysiology, 117, 2532-2539.

Sommer, W., Leuthold, H., Matt, J., 1998. The expectancies that govern the P300 amplitude are mostly automatic and unconscious. Behavioral and Brain Sciences, 21, 149-168.

Sommer, W., Matt, J., Leuthold, H., 1990. Consciousness of attention and expectancy as reflected in event-related potentials and reaction times. Journal of Experimental Psychology: Learning, Memory, and Cognition, 16, 902-915.

Squires, K.C., Petuchowski, S., Wickens, C., Donchin, E., 1977. The effects of stimulus sequence on ERPs: A comparison of visual and auditory sequences. Perception and Psychophysics, 22, 31-40.

Squires, N.K., Squires, K.C., Hillyard, S.A., 1975. Two varieties of long-latency positive waves evoked by unpredictable auditory stimuli in man. Electroencephalography and Clinical Neurophysiology, 38, 387-401.

Squires, K.C., Wickens, C., Squires, N.K., Donchin, E., 1976. The effects of stimulus sequence on the waveform of the cortical event related potential. Science, 193, 11421146.

Starr, A., Aguinaldo, T., Roe, M., Michalewski, H.J., 1997. Sequential changes of auditory processing during target detection: motor responding versus mental counting. 
Electroencephalography and Clinical Neurophysiology, 105, 201-212.

Steiner, G.Z., Barry, R.J., Gonsalvez, C.J., 2013a. Can working memory predict target-totarget interval effects on the P3? International Journal of Psychophysiology, 89, 399408.

Steiner, G.Z, Brennan, M.L., Gonsalvez, C.J., Barry, R.J., 2013b. Comparing P300 modulations: Target-to-target interval versus infrequent nontarget-to-nontarget interval in a three-stimulus task. Psychophysiology, 50, 187-194.

Stevens, M.C., Calhoun, V.D., Kiehl, K.A., 2005. fMRI in an oddball task: Effects of targetto-target interval. Psychophysiology, 42, 636-642.

Sutton, S., Braren, M., Zubin, J., John, E.R., 1965. Evoked potential correlates of stimulus uncertainty. Science, 150, 1187-1188.

Tabachnick, B.G., Fidell, L.S., 1989. Using multivariate statistics. New York: Harper Collins. Vaughan, H.G., Ritter, W., 1970. The sources of auditory evoked responses recorded from the human head. Electroencephalography and Clinical Neurophysiology, 28, 360-367.

Verleger, R., 1987. Sequential effects on P3 in a counting task: a partial replication. Biological Psychology, 25, 221-246.

Verleger, R., 1988. Event-related potentials and cognition: a critique of the context-updating hypothesis and an alternative interpretation of P3. Behavioral and Brain Sciences, 11, 343-356.

Verleger, R., 1991. The instruction to refrain from blinking affects auditory P3 and N1 amplitudes. Electroencephalography and Clinical Neurophysiology, 78, 240-251.

Verleger, R., 1997. On the utility of P3 latency as an index of mental chronometry. Psychophysiology, 34, 131-156.

Verleger, R., Jaśkowski, P., Wascher, E., 2005. Evidence for an integrative role of P3b in linking reaction to perception. Journal of Psychophysiology, 19, 165-181. 
Verleger, R., Möcks, J., 1987. Varimax may produce slow-wave-like shapes by merging monotonic trends with other components. Journal of Psychophysiology, 1, 265-270. Volpe, U., Mucci, A., Bucci, P., Merlotti, E., Galderisi, S., Maj, M., 2007. The cortical generators of P3a and P3b: A LORETA study. Brain Res Bull, 73, 220-230.

Watson, T.D., Azizian, A., Berry, S., Squires, N.K., 2005. Event-related potentials as an index of similarity between words and pictures. Psychophysiology, 42, 361-368. 


\section{Table Legend}

Table 1. F- and $p$-values, and partial effect sizes for the topographic analyses carried out on the two P300 components. 
Figure Legends

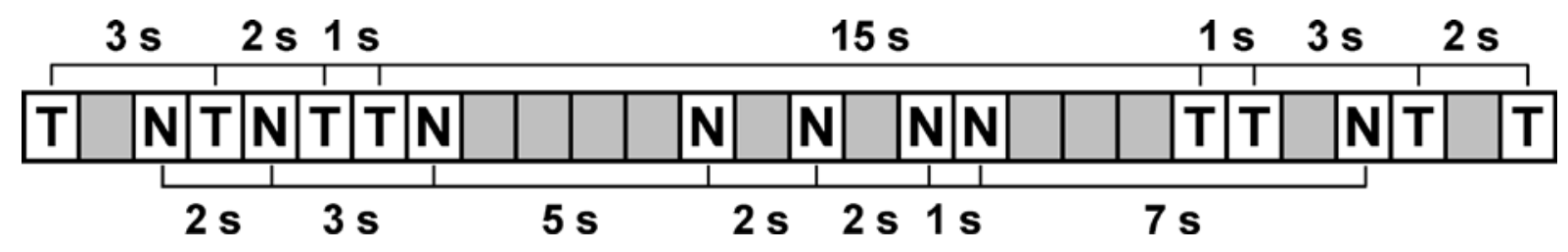

Figure 1. Illustration of the stimulus sequence including targets $(\mathrm{T})$, nontargets $(\mathrm{N})$, and silence (shaded rectangles). Each rectangle is indicative of the $1 \mathrm{~s}$ SOA. An example of seven sequential TTIs (3, 2, 1, 15, 1, 3, and 2 s) and NNIs (2, 3, 5, 2, 2, 1, and 7 s) are illustrated above and below, respectively. 


\section{Actual ERPs}
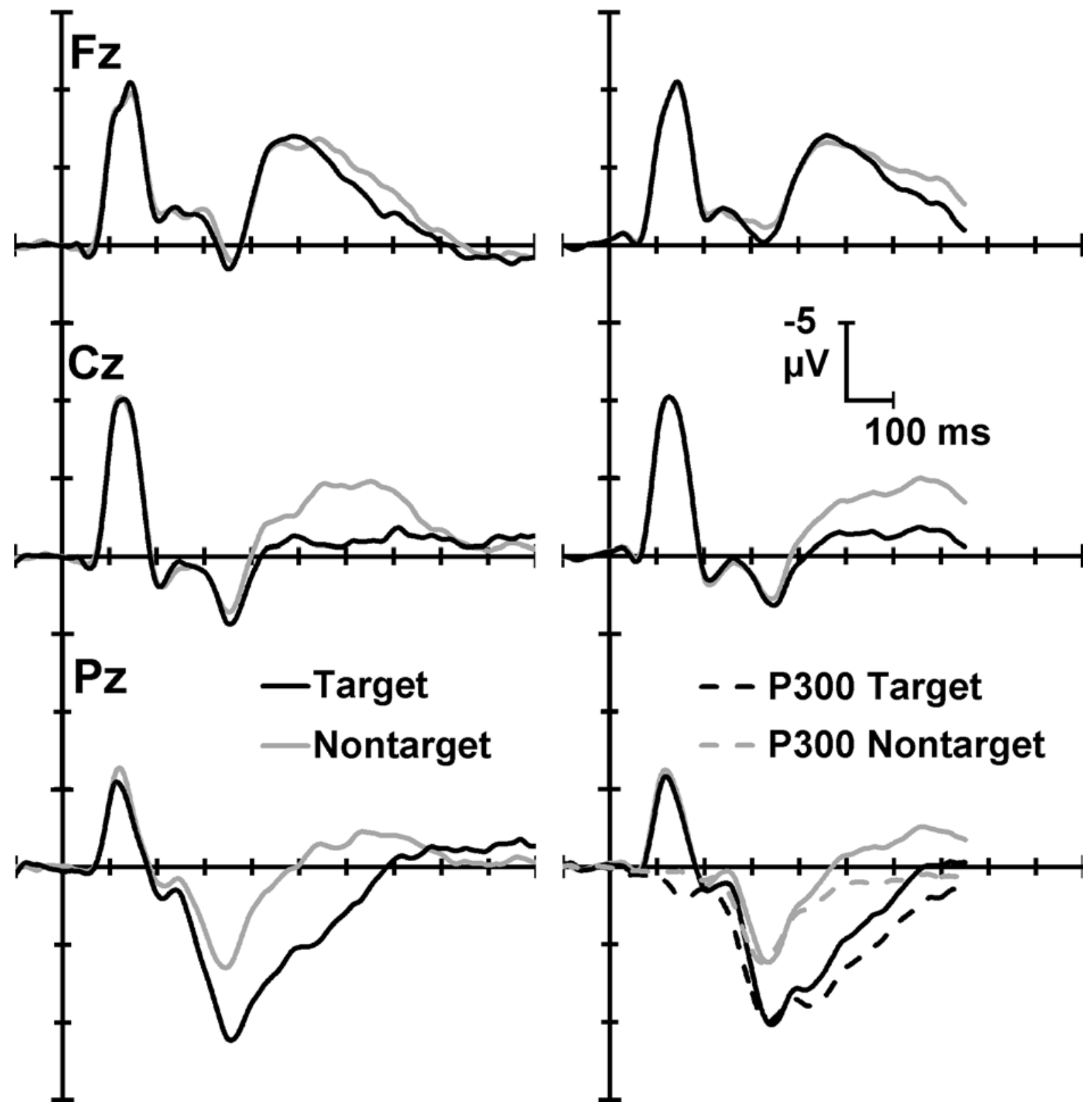

Figure 2. Mean (left) and corresponding PCA ERPs (right; derived from the sum of the seven temporal PCA factor scores) at midline sites for targets and nontargets. The dashed lines at Pz (maximal P300 site) represent the sum of the two PCA P300 components analysed (P3b, SW). 

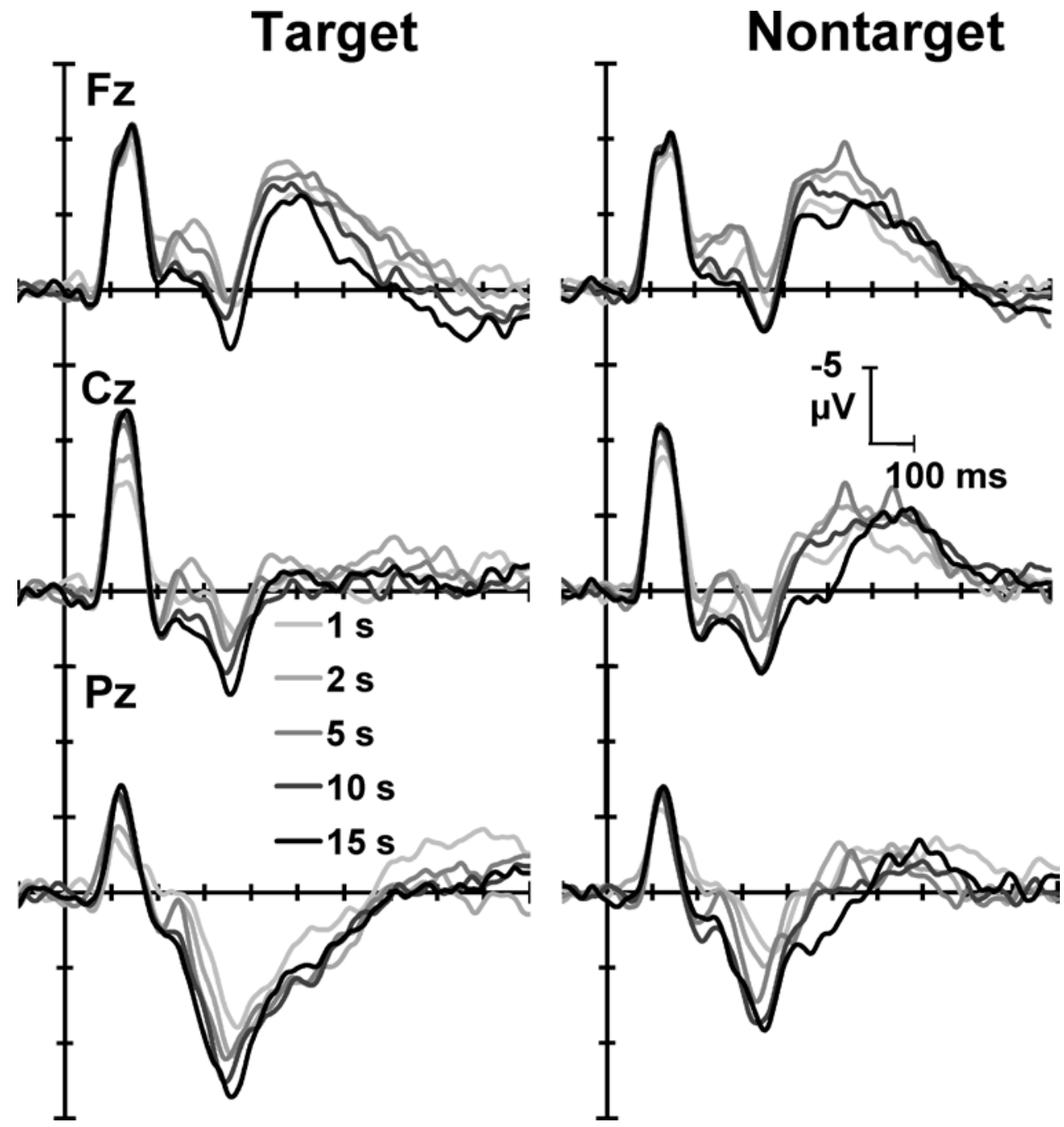

Figure 3. Mean ERPs at midline sites for targets (left) and nontargets (right) for each of the five intervals examined. Interval effects in the P300 component at Pz are clearly visible for both stimulus types. 

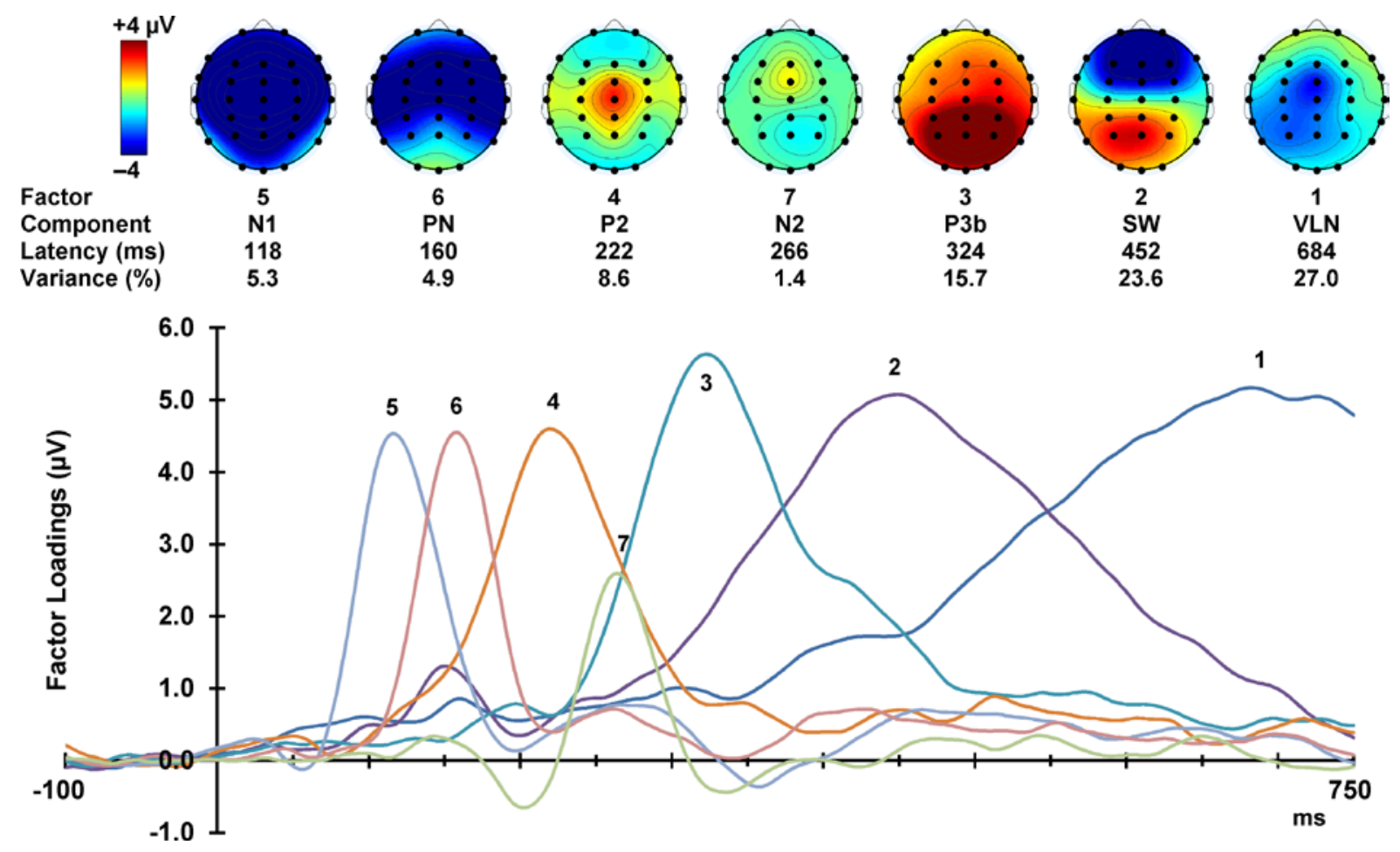

Figure 4. Upper: Topographic headmaps for each temporal PCA component averaged across all stimuli and intervals, with factor information below. Lower: Rescaled temporal PCA factor loadings as a function of time. 


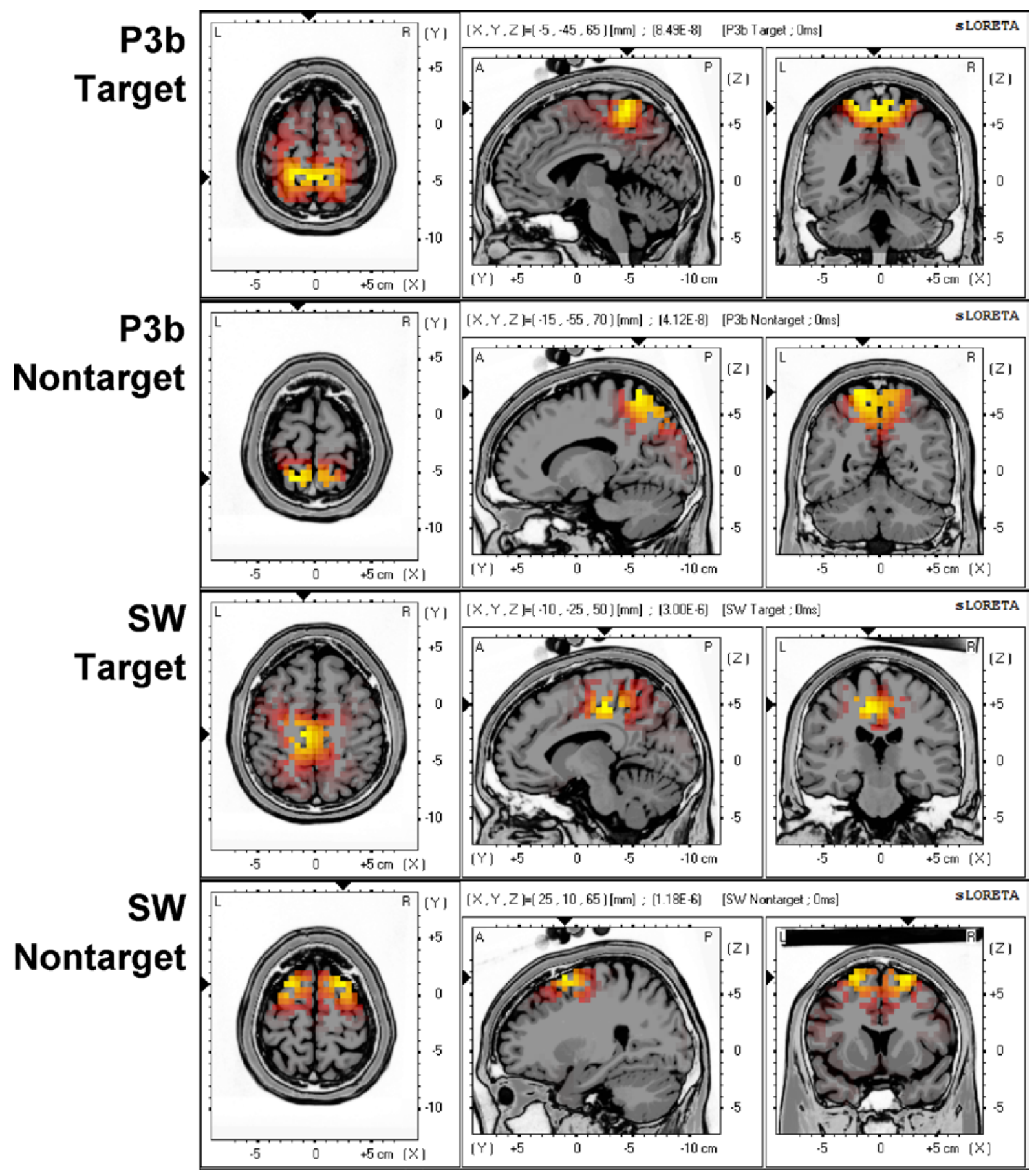

Figure 5. eLORETA images of the major sources for the PCA components identified as P3b and SW, separately for targets and nontargets. 

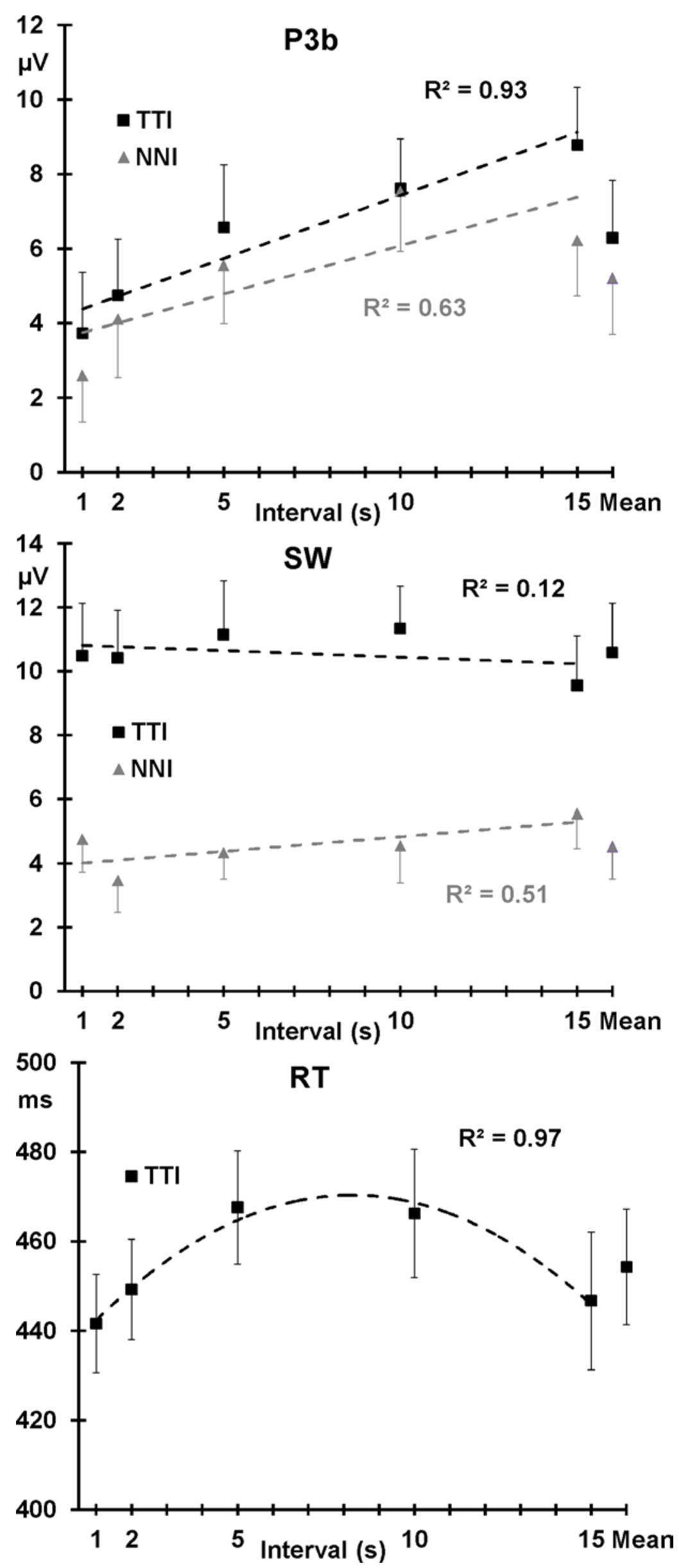
Figure 6. Upper and Middle: Target and nontarget means over intervals with standard error bars for P3b (top; mean of parietal sites), SW (middle; difference between means of parietal and frontal sites); dashed lines show linear trends, with the coefficient of determination indicated. Lower: Mean RT as a function of TTI with standard error illustrated; the dashed line indicates the quadratic trend, again with coefficient of determination. 